\title{
ESTRATEGIAS ADAPTATIVAS DEL SINDICALISMO ESPAÑOL CONTEMPORÁNEO
}

\author{
RAFAEL SERRANO DEL ROSAL
}

Instituto de Estudios Sociales Avanzados. CSIC. Córdoba.

PALABRAS CLAVE ADICIONALES

Crisis sindical, Movimiento obrero,

Sindicalismo contemporáneo, España.

\author{
ADDITIONAL KEYWORDS \\ Union Crisis, Worker Movement, \\ Contemporary Tradeunionism, Spain.
}

RESUMEN. En este artículo, el autor discute la tesis de la crisis u obsolescencia del sindicalismo en las sociedades industriales avanzadas, para el caso del sindicalismo español de finales del siglo XX. Para ello analiza, en primer lugar, la evolución reciente del mercado de trabajo español con objeto de describir el escenario en el que los trabajadores definen sus problemas y plantean sus demandas a los sindicatos, y, a su vez, el marco en el que los sindicatos definen sus objetivos y desarrollan estrategias para adaptarse y defender los intereses de los trabajadores; en segundo lugar, los problemas que consideran que tienen los trabajadores y las demandas que dirigen a los sindicatos, es decir, los intereses de los trabajadores; y, en tercer lugar, los principales objetivos sindicales y las estrategias que ponen en marcha para implementarlos en las postrimerías del siglo $\mathrm{XX}$. Con estos elementos se tratará de contestar a la pregunta: ¿se adaptan las organizaciones sindicales españolas de finales de siglo a los intereses de los trabajadores?.

\section{INTRODUCCIÓN}

El sindicalismo moderno, y sobre todo el que se ha denominado tradicionalmente de clase, tal y como hoy se conoce en la mayoría de los países industriales desarrollados, ha sido un actor característico del siglo XX. No obstante, en las últimos 20 años, el papel de las organizaciones sindicales ha empezado a

Revista Internacional de Sociología (RIS)

Tercera Época, nº 25, Enero-Abril, 2000, pp. 21-45. 
cuestionarse en distintos ámbitos. En el ámbito académico, se pueden encontrar multitud de referencias a la crisis, declive u obsolescencia de las organizaciones sindicales en diferentes países industrializados (Visser, 1992; Gallie, Penn y Rose, 1996; Ferner y Hymen, 1998; Goetschy, 1998; Instituto de Estudios y Análisis Económicos, 1993). Especialistas sobre el sindicalismo español, como Salvador Aguilar y Jordi Roca (1991), observan que la tesis de la crisis u obsolescencia del sindicalismo contemporáneo tiene numerosas variantes. Principalmente, destacan tres. La primera, sustentada por los que la atribuyen a la multiplicación de las reivindicaciones particulares y, por tanto, a la proliferación de diferentes formas de gremialismo. La segunda, refrendada por aquellos otros que consideran que la obsolescencia sindical se debe a la desaparición, o declive, de la fuerza de trabajo industrial clásica. Y la tercera, apoyada por los que consideran la incapacidad del sindicalismo para adaptarse a los cambios y defender los intereses reales de los trabajadores.

Dada la complejidad y variantes de la tesis de la crisis sindical, el presente artículo tiene como objetivo discutirla en un país y tiempo concretos -España, finales del siglo XX-, formulándola como la incapacidad de los sindicatos para adaptarse a los cambios y defender los intereses de los trabajadores. En otras palabras, en este artículo se trata de responder a la pregunta: ¿se adaptan las organizaciones sindicales españolas de finales de siglo a los intereses de los trabajadores?; o, en los términos anteriormente vistos, ¿están obsoletos los sindicatos españoles contemporáneos?

Para contestar ordenadamente a estas cuestiones se ha dividido el trabajo en tres partes, que corresponden a los tres apartados siguientes. En la primera, se analiza la evolución del mercado de trabajo español, desde mediados de los ochenta hasta la actualidad, con objeto de describir el marco objetivo en el que los trabajadores definen sus problemas y plantean sus intereses. Para ello, se ha utilizado, básicamente, información de la Encuesta de Población Activa, realizada por el Instituto Nacional de Estadística trimestralmente. En la segunda parte, se analizan los problemas que consideran que tienen los trabajadores españoles de los 90 y las demandas que éstos hacen a los sindicatos. Para ello, se ha utilizado, principalmente, el estudio 2088 del CIS — "Sindicatos"-, del que hemos realizado nuestra propia explotación. Y en la tercera parte se describen y analizan los principales objetivos y estrategias de los dos grandes sindicatos españoles de final de siglo: UGT y CC.OO. Ello con el fin de comprobar en qué medida se adecuan a los intereses de los trabajadores españoles - analizados en el apartado segundo-y, por tanto, poder responder a la cuestión de su obsolescencia. En esta tercera parte, se ha utilizado como material empírico una serie de entrevistas en profundidad a líderes sindicales de diferentes niveles organizativos de UGT y CC.OO., que el autor realizó para el estudio "European Trade Union and Change" financiado por la D.G. V de la Unión Europea', así como una serie de 15 grupos de discusión, compuestos o bien con trabajadores de diferentes secto- 
res, o bien con representantes sindicales de diferentes niveles organizativos, realizados para la investigación "Los problemas de integración sindical de las clases subordinadas en el contexto de la estructura social española de finales de siglo".

\section{EVOLUCIÓN DEL MERCADO DE TRABAJO ESPAÑOL DESDE MEDIADOS DE LOS 80}

Describir algunas de las características más importantes de la evolución del mercado de trabajo español, desde mediados de los 80 hasta la actualidad, tiene por objeto en este artículo detallar el escenario en el que los trabajadores definen sus problemas y plantean sus demandas a los sindicatos, y, a su vez, el marco en el que los sindicatos implementan sus objetivos desarrollando estrategias para adaptarse y defender los intereses de los trabajadores. Para ello, se va a comenzar analizando la relación existente, en dicho período, entre oferta y demanda de mano de obra².

En este análisis, lo primero que se observa es que, en los trece años que van de 1986 a 1999, se incrementa la oferta de mano de obra, es decir, hay más personas en disposición de trabajar. Concretamente, en este período, la población activa aumentó algo más de dos puntos porcentuales, pasando de represen-

\footnotetext{
1 Teniendo como base este informe, se han publicado dos obras que pueden ser de interés para el lector. Estas son: Rigby, M. y R. Serrano del Rosal (Coord.) (1997), Estrategias sindicales en Europa: convergencias o divergencias, Madrid, CES. Rigby, R., R. Smith y T. Lawlor (Eds.) (1999), European Trade Unions. Change and responses, London, Routledge.

${ }^{2}$ El concepto de mano de obra se ha utilizado y se utiliza en la literatura sociológica para referirse a poblaciones diferentes. Unas veces, se ha hecho referencia con el mismo a los trabajadores manuales, puesto que el concepto tiene su origen en otros períodos históricos en los que el obrero o trabajador manual era el componente más numeroso de la población asalariada. Otras se ha utilizado para hacer referencia a los trabajadores, manuales y no manuales, puesto que los primeros ya no son la mayoría. Con objeto de aclarar en qué sentido se utiliza en este trabajo de investigación hemos asumido la definición que se le da al mismo en el reciente trabajo de Martín Valverde, A. y J. García Murcia (1998), Glosario de empleo y relaciones laborales, Fundación Europea para la mejora de las condiciones del vida y de trabajo, Dublín, Irlanda. Lo utilizaremos como sinónimo del conjunto de la población activa cuando nos referimos al mercado de trabajo, y como trabajador, manual o no, cuando nos referimos a condiciones laborales, legislación etc. Concretamente los autores citados lo definen como sigue: "MANO DE OBRA: expresión que utilizada en sentido macroeconómico se refiere al conjunto de la población activa; desde el punto de vista empresarial, se circunscribe al factor trabajo como uno de los factores de producción (...) cada vez se asocia más al término trabajador. En la legislación laboral y de empleo se usa como sinónimo de trabajadores".
} 
tar el 47,7\% de la población española de 16 años o más en 1986, al 49,9\% en 1999.

Por otra parte, si se observa el comportamiento de la demanda de mano de obra —oferta de puestos de trabajo-, se encuentra, que hasta 1991, aumentó por encima del incremento de la población activa, por lo que la tasa de paro llegó a reducirse en este período hasta 5 puntos, siendo en 1986 del 21,5\% y, en 1991, del $16,3 \%$.

Pero, a partir de 1992, con el cambio de ciclo económico, la demanda de mano de obra vuelve a descender, con el consiguiente incremento del paro, que llegó a alcanzar en 1997 una tasa similar a la que había en 1986, esto es, de alrededor del $21 \%$. Posteriormente, con el nuevo cambio de ciclo vuelve a descender levemente, situándose en 1999 en una tasa cercana al 17\%.

Atendiendo, por tanto, a las cifras globales de oferta y demanda de mano de obra, es verosímil concluir que, en general, el mercado de trabajo español ha sido capaz de absorber las nuevas entradas en el mismo, es decir, ha sido capaz de crear empleo en volumen similar, al menos, al incremento de la población activa. Pero, igualmente, se ha de subrayar que no ha generado la suficiente oferta de trabajo como para reducir, significativamente, la alta tasa de paro que afecta a la población activa española.

La existencia de esta elevada tasa de paro sustentaría el hecho de que los españoles, en diferentes encuestas de opinión realizadas en este período de tiem$\mathrm{po}^{3}$, hayan considerado el desempleo como el problema más importante que afecta a la sociedad española o, cuando menos, uno de los tres más importantes.

No obstante, para profundizar más en el conocimiento del marco objetivo en el que se fraguan los intereses de los trabajadores españoles en el último decenio, se debe desagregar más la información aportada sobre el mercado de trabajo e introducir en el análisis algunas variables, como por ejemplo, el género o la edad. De esta forma se puede observar que, mientras que la tasa de actividad masculina descendió casi siete puntos en el período de referencia —es decir, un menor número de hombres se incorporaron al mercado de trabajo-, la femenina aumentó más de nueve, lo cual permite afirmar que el incremento en la oferta de mano de obra que se ha constatado anteriormente se debe, principalmente, a la

\footnotetext{
${ }^{3}$ Esta información se puede contrastar por ejemplo con los diferentes estudios de opinión realizados por el CIS en los que se ha incluido alguna pregunta igual o similar a la que sigue: "De los siguientes temas que voy a mencionarle, ¿cuáles cree $\mathrm{Vd}$. que actualmente preocupan más a los españoles?.

01. El funcionamiento de los servicios públicos; 02. La protección del medio ambiente 03. La vivienda 04 . La inmigración, es decir, la llegada de trabajadores procedentes de países menos desarrollados; 05. La droga; 06. La delincuencia; 07. El paro; 08. La situación política; 09. La conflictividad laboral; 10. El mantenimiento del nivel de vida; 11. Otros ¿cuál?...
} 
incorporación de las mujeres al mercado de trabajo ${ }^{4}$. De hecho, en términos relativos, el número de hombres que forman parte de la población activa española disminuyó en dicho período, lo que en parte explica que la tasa de paro masculina se redujese casi ocho puntos (del 20,4\% en 1986 al 12,4\% en 1999), mientras que la femenina aumentase hasta seis puntos a mediados de los 90 , para posteriormente situarse en tasas similares a las habidas en 1986 (25,4\% en 1986, 31,4\% en 1995 y $25,3 \%$ en 1999).

Con la información expuesta se quiere subrayar que, a pesar de que la tasa de paro española, desde mediados de los 80 hasta la actualidad, se sitúa siempre por lo alto del 16\%, el desempleo no ha afectado en todo el período al mismo tipo de personas, ya que éste se atenúa entre la población activa masculina y se acentúa, en términos relativos, entre la población activa femenina.

Otra variable que introducida en el análisis clarifica las cifras globales aportadas sobre el mercado de trabajo español en el período 1986-99, es la edad.

Si bien es cierto que en el período antes mencionado aumentó en dos puntos la oferta de mano de obra, también lo es que este incremento se concentró en el intervalo de edad que va de los 25 a los 54 años, puesto que, en todos los demás intervalos de edad, la población activa disminuyó considerablemente en términos relativos.

Dicho descenso es especialmente importante entre los más jóvenes, es decir, en la población de 16 a 19 años. En este intervalo de edad, la población activa era en 1986 del 37,1\% y, en 1999, de sólo el 24,3\%.

Aunque no es objeto de este artículo profundizar en el análisis de dicho fenómeno ${ }^{5}$, merece la pena ponerlo en relación con otro que, al menos, correlaciona con aquél. Me refiero a la mayor permanencia de los jóvenes españoles dentro del sistema educativo, que provoca, a su vez, cierto retraso en la edad media con la que los españoles se incorporan al mercado de trabajo. No obstante, ha de apuntarse también que este retraso está influido por las altas tasas de paro que soportan los activos de dicho grupo de edad. Concretamente, en el intervalo de edad que va de los 16 a los 19 años, la tasa de paro se mantiene, en todo este período, en cotas muy superiores a las que se dan en los demás intervalos de

\footnotetext{
${ }^{4}$ Sobre la evolución del mercado de trabajo en general y sobre la situación de la mujer en éste, en particular, se puede consultar Toharia, L. (Dir.)(1994), "Empleo y paro" en V Informe sociológico de la situación social de España, Sociedad para todos en el año 2000, Madrid, Fundación Foessa.

${ }^{5}$ Para ello se pueden consultar trabajos específicos como el capítulo anteriormente citado, sobre empleo y paro, dirigido por Luis Toharia (1994). O, también el libro de Sanchís E. (1991), De la escuela al paro, Siglo XXI, Madrid.
} 
Tabla 1.

Tasas de actividad y paro por edades

\begin{tabular}{|c|c|c|c|c|c|c|c|c|}
\hline \multirow[b]{3}{*}{ Años } & \multicolumn{8}{|c|}{ INTERVALOS DE EDAD EN AÑOS } \\
\hline & \multicolumn{2}{|c|}{$16-19$} & \multicolumn{2}{|c|}{$20-24$} & \multicolumn{2}{|c|}{$25-54$} & \multicolumn{2}{|c|}{$55 y+$} \\
\hline & $\mathrm{A}$ & $P$ & A & $\mathrm{P}$ & $\bar{A}$ & $\mathrm{P}$ & $\mathrm{A}$ & $\mathrm{P}$ \\
\hline 1986 & 36,3 & 52,3 & 62,3 & 44,2 & 64,7 & 15,3 & 21,2 & 10,7 \\
\hline 1987 & 38,5 & 49,5 & 66,0 & 40,4 & 66,6 & 15,0 & 20,5 & 9,3 \\
\hline 1988 & 37,2 & 45,7 & 68,2 & 37,3 & 68,1 & 14,7 & 20,0 & 8,4 \\
\hline 1989 & 33,8 & 38,2 & 67,2 & 32,8 & 69,0 & 13,7 & 20,0 & 8,2 \\
\hline 1990 & 32,3 & 36,6 & 67,2 & 30,6 & 70,2 & 13,1 & 19,5 & 7,6 \\
\hline 1991 & 30,1 & 34,7 & 66,1 & 29,7 & 71,0 & 13,7 & 18,9 & 8,0 \\
\hline 1992 & 29,8 & 38,9 & 63,9 & 32,7 & 71,4 & 15,7 & 18,5 & 9,0 \\
\hline 1993 & 28,5 & 50,3 & 63,0 & 40,5 & 72,4 & 19,4 & 17,7 & 10,8 \\
\hline 1994 & 27,0 & 53,1 & 62,8 & 42,3 & 73,4 & 20,9 & 16,4 & 11,6 \\
\hline 1995 & 25,1 & 50,6 & 61,3 & 39,8 & 73,9 & 20,0 & 16,0 & 11,4 \\
\hline 1996 & 24,3 & 50,8 & 59,9 & 39,3 & 74,6 & 19,3 & 16,0 & 10,9 \\
\hline 1997 & 24,0 & 50,9 & 59,1 & 35,5 & 75,2 & 18,2 & 15,9 & 10,8 \\
\hline $1998^{1}$ & 23,2 & 47,2 & 58,4 & 29,4 & 75,3 & 17,2 & 15,9 & 10,0 \\
\hline $1999^{1}$ & 24,3 & 40,7 & 59,2 & 26,7 & 75,7 & 15,0 & 15,4 & 9,2 \\
\hline
\end{tabular}

$\mathrm{A}=$ Activos (\% sobre población mayor de 16 años).

$\mathrm{P}=$ Parados (\% sobre población activa).

' Datos del primer trimestre.

Fuente: Encuesta de población activa, INE.

edad. Por ello, la opción de abandonar el sistema educativo e incorporarse al mercado de trabajo se posterga en muchos casos, valorando la relación positiva existente entre nivel de formación y probabilidad de encontrar trabajo ${ }^{6}$.

Por último, para esta descripción del mercado de trabajo español, como marco en el que los trabajadores definen sus intereses, es significativo analizar la situación profesional de aquella parte de la población activa que está ocupada. Con ello se centrará el análisis en aquellas personas que son la base objetiva de referencia de los sindicatos, los trabajadores de hecho. Atendiendo a esta variable encontramos dos tendencias dignas de subrayar. La primera, el incremento en términos relativos del número de asalariados. Concretamente, la proporción

"Sobre la relación entre educación y trabajo en la España de los 90 se puede consultar Miguel, A. de (1992), "Educación y Trabajo", en La sociedad española, 1992-1993, Madrid, Alianza Editoral. 
de asalariados sobre el total de ocupados aumentó en España un 13\% en el período 1986-99, lo cual tiene como consecuencia que, al final del período, representen más de las tres cuartas partes de la población española ocupada, concretamente el $77,8 \%$.

La segunda tendencia es el crecimiento constante y, en términos relativos, espectacular, del número de empleadores (empresarios), que crece más del $94 \%$ en dicho período, representando en 1999 el 5,4\% de la población ocupada española.

Si se analizan ambas tendencias conjuntamente, puede verse cómo el crecimiento relativo de los empresarios ha sido superior al de los asalariados, lo que ha tenido como consecuencia el incremento del número de empresas que hay en España y la reducción de su tamaño medio. De hecho, se puede comprobar cómo ya a mediados de los 90 , alrededor del $98 \%$ de las empresas españolas tenía menos de 50 trabajadores, es decir, eran pequeñas empresas; cifras que son aún más significativas cuando se comprueba que, en esas fechas, más de las tres cuartas partes de estas empresas tenían cinco trabajadores o menos, lo que constituye una característica del mercado de trabajo español que, como se verá en el epígrafe cuarto de este artículo, los sindicatos tienen en cuenta, tanto en la definición de sus objetivos, como en la implementación de sus estrategias de adaptación y defensa de los intereses de los trabajadores contemporáneos.

En suma, los datos aportados indican que, en el período 1986-99, se incrementó la oferta de mano de obra en el mercado de trabajo español, principalmente por la mayor incorporación de la mujer. El desempleo se mantuvo en cotas muy superiores a las que había en los demás países de su entorno, independientemente de la bonanza o crisis de ciclo económico. Además, esta situación de exclusión afectó en mayor medida a las mujeres que a los hombres, concentrándose también más entre la población más joven, que, a pesar de retrasar su entrada en el mercado de trabajo, siguió siendo el grupo de edad más afectado por el desempleo. Y, todo ello, en un marco empresarial dominado cada vez más por la pequeña empresa o, incluso, por la micro-empresa (de 5 o menos trabajadores).

Las citadas características de la evolución reciente del mercado de trabajo español se han traído a colación en este artículo porque influyen, directa o indirectamente, tanto en la configuración de los intereses - problemas y demandas - de los trabajadores españoles contemporáneos, como se verá en el siguiente apartado, como en los principales objetivos de los sindicatos españoles y en las estrategias que éstos ponen en marcha para alcanzarlos, como se verá en el epígrafe cuarto.

\section{UNA APROXIMACIÓN A LOS INTERESES DE LOS TRABAJADORES}

Un segundo elemento a tener en cuenta en el argumento central de este artículo es el relativo a los problemas que consideran que tienen los trabajadores de 
los 90 y las demandas que éstos dirigen a los sindicatos ${ }^{7}$, toda vez que serán éstos los que, en conjunto, nos permitan conocer cuáles son sus intereses; lo cual, junto con el análisis de los objetivos y estrategias de los grandes sindicatos, que se hará posteriormente, nos permitirá, según el argumento expuesto en la introducción de este artículo, discutir la tesis de la obsolescencia o crisis de los sindicatos en las sociedades industriales avanzadas, para el caso de España en las postrimetrías del siglo XX.

Una primera observación es que más de las tres cuartas partes $(79,2 \%)$ de la población ocupada están satisfechas o muy satisfechas con su trabajo, lo cual se comprende mejor si no se olvida que dicha opinión se produce en un mercado de trabajo que cuenta, como se ha visto en el apartado anterior, con una tasa estructural de desempleo superior al $15 \%$ de la población activa, lo que sin duda influye en que sólo el hecho de tener empleo ya es motivo de satisfacción. No obstante, los principales motivos que aducen los trabajadores que están satisfechos o muy satisfechos con su trabajo son los siguientes: el ambiente de trabajo, la estabilidad en el empleo, el tipo de trabajo que realizan y el salario que reciben; mientras que los motivos que esgrimen los trabajadores que no están satisfechos con su trabajo actual son, principalmente, el bajo salario que perciben, su inestabilidad en el empleo y las malas condiciones de trabajo.

Esta información nos lleva a concluir que, en la España de los noventa, la satisfacción con el empleo — de aquéllos que lo tienen—está directamente rela-

Tabla 2.

Satisfacción con el trabajo.

\begin{tabular}{lrr}
\hline & Frecuencia & $\%$ \\
\hline Muy satisfecho & 586 & 20,4 \\
Satisfecho & 1686 & 58,8 \\
Ni satisfecho ni insatisfecho (no leida) & 230 & 8,0 \\
Insatisfecho & 296 & 10,3 \\
Muy insatisfecho & 61 & 2,1 \\
No sabe & 1 & 0,0 \\
No contesta & 8 & 0,3 \\
Total & 2868 & 100 \\
\hline
\end{tabular}

Fuente: Elaboración propia a partir de los datos del estudio 2088 del CIS.

\footnotetext{
7 Para ello se utilizarán las opiniones recogidas en el estudio 2088 del CIS, "Sindicatos", de mayo-julio de 1994.
} 
Tabla 3.

Razones para la satisfacción con el trabajo.

\begin{tabular}{lcc}
\hline & Frecuencia & \% de respuestas \\
\hline Salario bueno & 629 & 14,3 \\
Empleo estable & 759 & 17,2 \\
Oportunidades de ascenso & 87 & 2,0 \\
Buenas condiciones de trabajo & 609 & 13,8 \\
Ambiente agradable & 934 & 21,2 \\
Oportunidades de formación & 107 & 2,4 \\
Trabajo interesante & 654 & 14,9 \\
Permite realizarse profesionalmente & 447 & 10,2 \\
Otra razón & 155 & 3,5 \\
No sabe & 11 & 0,2 \\
No contesta & 10 & 0,2 \\
\hline
\end{tabular}

Fuente: Elaboración propia a partir de los datos del estudio 2088 del CIS.

Tabla 4.

Razones para la no satisfacción con el trabajo.

\begin{tabular}{lcc}
\hline & Frecuencia & $\%$ de respuesta \\
\hline Salario bajo & 151 & 25,0 \\
Empleo inestable & 101 & 16,7 \\
No oportunidades de ascenso & 45 & 7,4 \\
Malas condiciones de trabajo & 80 & 13,2 \\
Mal ambiente & 41 & 6,8 \\
No hay oportunidades de formación & 35 & 5,8 \\
Trabajo aburrido & 45 & 7,4 \\
No permite realizarse profesionalmente & 58 & 9,6 \\
Exceso de trabajo & 8 & 1,3 \\
Otra razón & 37 & 6,1 \\
No sabe & 3 & 0,5 \\
No contesta & 1 & 0,2 \\
\hline
\end{tabular}

Fuente: Elaboración propia a partir de los datos del estudio 2088 del CIS.

cionada con la estabilidad del mismo, las condiciones de trabajo y el salario. Hecho que cobra mayor verosimilitud cuando lo contrastamos con la información sobre el mercado de trabajo que se ha presentado en el apartado anterior; es decir, cuando se conoce que dicha percepción se produce en un mercado de trabajo que cuenta con una alta tasa de desempleo y en el que la mayor parte de 
las empresas son pequeñas, con lo que esto supone para la estabilidad, el salario y las condiciones de trabajo.

La preocupación de los trabajadores por los temas mencionados se traduce en demandas, explícitas o implícitas, que dirigen a los sindicatos. Éstas se han medido utilizando como indicador un ítem, del estudio 2088 del CIS, que preguntaba a los trabajadores sobre los temas principales que consideraban que deberían negociar los sindicatos. Las respuestas más frecuentes eran, por orden de importancia, las condiciones de trabajo, los tipos de contrato, las bandas salariales y las formas de protección ante el desempleo. Por tanto, aquello que los trabajadores demandan a los sindicatos, sus intereses, está en sintonía con lo que ellos consideran que son los problemas laborales que más les afectan. $\mathrm{O}$ dicho de otra forma, los trabajadores justifican el papel de los sindicatos en la medida en que éstos sean capaces de resolver, o al menos negociar y defender, lo que entienden que son sus intereses. Ello no significa que los trabajadores no consideren importante el papel de las organizaciones sindicales. De hecho, más del $60 \%$ de los encuestados consideraba, a mediados de los 90 , que los sindicatos son fundamentales para proteger sus intereses, pero ratificándose en la opinión de que no deben intervenir en política y deben limitarse a los problemas laborales, postura con la que estaban de acuerdo más del $67 \%$.

En este sentido, se detecta un importante cambio de opinión, en relación a lo que se opinaba a principios de los 80 . En aquella época, en un conocido estudio dirigido por Robert Fishman" en colaboración con el CIS, ante la pregunta de si los sindicatos deberían dedicarse exclusivamente a defender a los trabajadores, o si también deberían participar activamente en la política, la economía y los problemas sociales del país, más del $70 \%$ de los encuestados eligieron la segunda opción.

Este cambio de intereses sustenta la interpretación de que, en los años 90, los trabajadores circunscriben el papel de las organizaciones sindicales al plano laboral, dejando su dimensión política global, cuando menos, en un segundo plano. De hecho, como se ha visto, los trabajadores justifican actualmente el papel de los sindicatos en la medida en que éstos sean capaces de resolver, o al menos negociar y defender, sus propios intereses. Es decir, la lógica con la que valoran a los sindicatos es instrumental y pragmática, teniendo un papel secundario la lógica ideológica-identitaria que sustentó a las organizaciones sindicales espa-

\footnotetext{
${ }^{8}$ Sobre la aproximación de los sindicatos a las pequeñas y medianas empresas se puede consultar el artículo Blanco J. (1991), «La sindicación de trabajadores de pequeñas y medianas empresas», Sociología del Trabajo, num. 11.

'El estudio empírico es el 1278b (mayo-junio 1981) del CIS. Sobre este cuestionario hay una explotación realizada por el propio Fishman y recientemente publicada por el CIS.
} 
ñolas en la época postfranquista, lo cual no es un fenómeno exclusivo de España, sino más bien una tendencia en el conjunto del sindicalismo europeo ${ }^{10}$.

Cuando se analiza su opinión sobre cuál creen que es el nivel de preocupación de los sindicatos por defender los intereses de diferentes colectivos de trabajadores, como las mujeres, los jóvenes o los parados, es decir, aquellos que se ha visto que están en peores condiciones en el mercado laboral español, es interesante comprobar cómo la mayoría considera que los sindicatos se preocupan poco o nada de estos colectivos, excepto del colectivo de trabajadores con trabajo seguro, que, aunque por un estrecho margen, la mayoría de los encuestados opinaba que son objeto de mucha o bastante preocupación sindical (gráfico 1).

La opinión puesta de manifiesto sobre la falta de preocupación de los sindicatos por defender los intereses de todos aquéllos que no tengan un "trabajo seguro", ayuda a comprender que el $54 \%$ de los encuestados opine que los sindicatos son, actualmente, organizaciones desfasadas que deberían de cambiar; frente a sólo un $30 \%$ que los considera organizaciones modernas y adaptadas a la reali-

Gráfico 1 .

Opinión sobre la preocupación de los sindicatos en la defensa de diferentes colectivos.

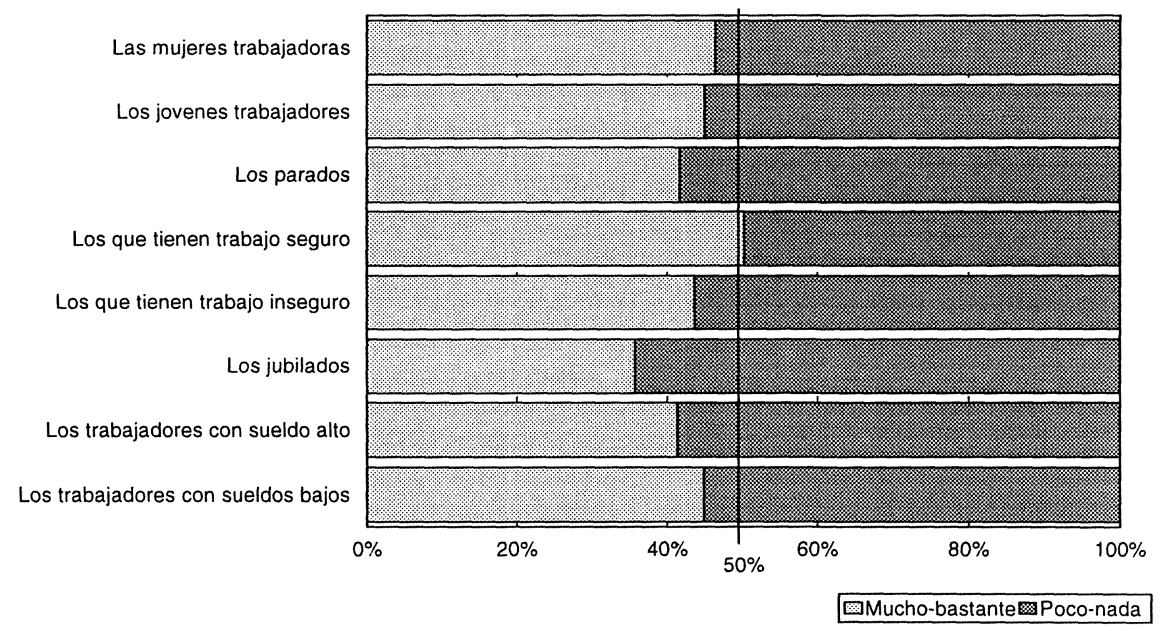

Fuente: Elaboración propia a partir de estudio 2088 del CIS.

10 Sobre dicho fenómeno puede consultarse el libro colectivo coordinado por Rigby, M. y R. Serrano del Rosal (1997), Estrategias sindicales en Europa: convergencias o divergencias, CES, Madrid. 
dad. Además justifica que se plantee la discusión sobre la crisis u obsolescencia sindical en los términos que lo hace este trabajo, a saber, evaluando si los objetivos y las estrategias sindicales se adaptan a los intereses de los trabajadores en un país y tiempo concretos - España finales del siglo XX.

En conjunto, estos y otros considerandos subjetivos sobre la realidad sindical, dan cuenta de la imagen que la población trabajadora tiene sobre el sindicalismo español contemporáneo, lo cual tiene su reflejo directo, por ejemplo, en que los trabajadores comiencen a valorar, en porcentajes importantes, nuevas vías no sindicales de protección de sus derechos y condiciones laborales. De tal forma, que analíticamente se podrían construir dos grandes grupos. Por un lado, el que forman aquellos trabajadores que optan por defender sus intereses en la empresa a través de los representantes sindicales, es decir, los que consideran que la acción colectiva encauzada por los sindicatos es la mejor forma de defender sus propios intereses. Y por otro, aquéllos que valoran más positivamente la acción individual, ya sea a través de las influencias personales, ya sea a través de un abogado.

Tabla 5.

Mejor forma de defender los problemas que le surgen en la empresa.

\begin{tabular}{lcc} 
& Frecuencia & \% de respuestas \\
\hline A través de los representantes de los trabajadores & 1117 & 39,0 \\
Por mi cuenta, a través de un abogado & 762 & 26,6 \\
Con influencias personales en la empresa & 369 & 12,9 \\
Otras & 277 & 9,7 \\
No sabe & 237 & 8,3 \\
No contesta & 103 & 3,6 \\
Total & 2865 & 100 \\
\hline
\end{tabular}

Fuente: Elaboración propia a partir de los datos del estudio $2088 \mathrm{del}$ CIS.

En suma, tenemos que la mayoría de la población en edad de trabajar, sean trabajadores en ese momento o no, ante un mercado de trabajo como el que se ha descrito, considera que los sindicatos deberían de modernizarse adaptándose, más y mejor, a la nueva realidad del mundo del trabajo, lo cual pasaría por un mayor conocimiento y contacto con los trabajadores en particular, y con la población en edad de trabajar en general, y por adoptar estrategias de actuación realistas que defiendan sus intereses, a saber, que mejoren las condiciones de trabajo, la estabilidad del mismo y el mantenimiento de un salario digno. 


\section{OBJETIVOS Y ESTRATEGIAS SINDICALES ${ }^{11}$}

En los dos epígrafes anteriores se ha descrito la situación del mercado de trabajo español desde mediados de los 80 , los principales problemas de los trabajadores y las demandas que éstos plantean a los sindicatos en las postrimerías del siglo XX. En este otro se expondrán los objetivos de los grandes sindicatos españoles -UGT y CC.OO. - y las estrategias más importantes que durante dicho período han puesto en marcha para implementarlos. Con ello, se tendrán los elementos necesarios para discutir, en la España contemporánea, la tesis de la obsolescencia de las organizaciones sindicales, sobre la base de si existe o no una adecuación entre las estrategias sindicales y los intereses - problemas y demandas- de los trabajadores. No obstante, antes de comenzar con dicha tarea, consideramos necesario delimitar el concepto de estrategia sindical que se utilizará en adelante y explicar la estrategia de análisis que se seguirá en este apartado.

Se ha optado por utilizar una definición de estrategia sindical lo suficientemente amplia como para que cubra el espacio que va desde la estrategia stricto $s e n s u$, a la respuesta sindical informada ${ }^{12}$. Además, por razones de claridad en el análisis hemos considerado oportuno exponer conjuntamente estrategias y objetivos sindicales. No se ha optado por exponer de forma separada los objetivos sindicales y las estrategias, puesto que los objetivos que tratan de alcanzar los sindicatos, con una o varias estrategias, sólo cobran sentido si se ponen en relación con el desarrollo de otra u otras estrategias.

\footnotetext{
"La información que se expondrá en este apartado se ha obtenido, principalmente, mediante la explotación de 5 entrevistas no estructuradas y 47 semiestructuradas a líderes sindicales españoles de diferentes niveles organizativos y distintos sectores productivos de UGT y CC.OO. Dichas entrevistas, realizadas por el autor, se hicieron durante los años 1995 y 1996 en el marco de una investigación financiada por la Dirección General V de la U.E. y, en el caso de España, con fondos propios del IESA de Andalucía/CSIC, titulada European Trade Union and Change. Sobre las características técnicas de dicho trabajo se puede consultar Serrano del Rosal, R. (1997) "El diseño de la investigación" en Rigby, M. y R. Serrano del Rosal (Coord.), Estrategias sindicales en Europa convergencias o divergencias, Madrid, Consejo Económico y Social.

${ }^{12}$ En este sentido asumimos las recientes aportaciones que diferentes autores han hecho al concepto clásico de estrategia, con objeto de aplicarlo al estudio de la acción sindical. Por ejemplo, para Leisink las estrategias no tienen por qué implicar por sí mismas un proceso racional de estimación entre diferentes alternativas, ni una continuidad consciente, desde el diseño hasta su implementación. La racionalidad no obedece a una estimación a largo plazo, sino que a menudo se ve muy limitada y centrada en una decisión inmediata. Además, los desacuerdos internos pueden generar intentos por evitar la implementación de decisiones ya adoptadas (Leisink, 1996). O también Undy y otros relajan este concepto, cuando explican cómo las estrategias son interpretadas a partir del comportamiento sindical. Puede suceder, por tanto, que en realidad no sean estrategias en sí mismas en el sentido de que hayan sido diseñadas de forma racional como un conjunto de políticas integrado homogéneo y coherente (Undy, 1996).
} 


\section{Especialización vertical y penetración en la pequeña empresa}

Una de las principales estrategias de los grandes sindicatos españoles de finales de siglo, ha sido la especialización vertical de sus estructuras, con la que han tratado de alcanzar diferentes objetivos. El primero, capacitar a sus organizaciones para dar mejor respuesta a las peculiaridades de cada sector productivo. Es decir, conseguir unas estructuras sindicales más especializadas e informadas, que no sólo tengan capacidad de respuesta ante los movimientos empresariales, sino también la facultad de análisis suficiente como para abordar los difíciles procesos de negociación que se plantean en las sociedades industriales avanzadas contemporáneas, con lo que tratan de adaptarse estructuralmente para defender mejor las condiciones laborales de los trabajadores. El segundo objetivo de esta estrategia ha sido desterrar su tradicional actitud pasiva-reactiva, en favor de una actitud activa y con capacidad de presentar alternativas innovadoras y realistas para cada sector productivo. Y el tercero, resultado de los dos anteriores, ha sido preservar, en la medida de lo posible, otro de los principales intereses de los trabajadores como es la estabilidad en el empleo, y borrar la imagen confrontadora y de freno a la expansión empresarial que muchos empresarios atribuyen a las organizaciones sindicales.

Para ello han puesto en marcha un doble proceso de reestructuración. Por una parte, la potenciación económica y organizativa de las estructuras de rama, y por otra, la fusión de las estructuras sindicales de industrias afines ${ }^{13}$ con objeto de fortalecerlas y capacitarlas para afrontar problemas comunes maximizando los recursos. Con estos cambios han intentado acercar el sindicato a los trabajadores, mostrando su conocimiento de los problemas concretos que más les afectan, así como su especialización y capacidad para resolverlos.

Esta estrategia sindical estaría estrechamente relacionada con otra, como es la de penetración en la pequeña empresa. Los sindicatos españoles conocen de sus deficiencias en este tipo de empresas y, a lo largo de los 90 , han intentado desarrollar diferentes estrategias para penetrar en ellas y, así, conseguir el apoyo de sus trabajadores que, generalmente, tienen mayor inestabilidad y peor salario que los de las grandes empresas. Esta estrategia se ha concretado en algunas acciones, como la de utilizar las campañas de promoción de las elecciones sindi-

\footnotetext{
${ }^{13}$ Este tipo de fusiones se han producido en ambos sindicatos. Como ejemplo de las muchas fusiones que se han producido en los últimos años podemos citar, en UGT, la fusión de los sindicatos del metal, la construcción y las industrias afines en la M.C.A. o la fusión de química y energía, textil, piel y minería en la FIA; y, en CC.OO, la fusión de la federación del espectáculo, cultura y deportes, con la federación del papel, artes gráficas y comunicación social en la FESPACE y posteriormente la fusión de ésta con la de transporte comunicaciones y mar (FETCOMAR).
} 
cales para establecer un primer contacto con los trabajadores de este tipo de empresas (Jordana, 1994:155). Sin embargo, este camino no ha resultado muy efectivo, principalmente por la dificultad de encontrar en este tipo de empresas trabajadores en condiciones de enfrentarse a una cultura empresarial que, generalmente, ve como una agresión que los trabajadores se sindiquen y defiendan sus intereses colectivamente. Ello ha motivado que los sindicatos desarrollen otras acciones encaminadas a afrontar dichos problemas, sin necesidad de enfrentar, directamente, a los trabajadores con sus empleadores. Ello se ha intentado, por ejemplo, con la utilización de liberados sindicales ${ }^{14}$ que tienen como misión, en primer lugar, visitar las pequeñas empresas de un mismo sector y zona, con objeto de conocer los problemas de los trabajadores, $y$, en segundo lugar, recabar su apoyo para que deleguen en ellos el seguimiento de sus convenios colectivos y la negociación de sus intereses. Esta acción parece acertada por los resultados positivos que ha dado donde se ha puesto en marcha. Empero, se ve frenada por la escasez de recursos que los sindicatos pueden dedicar a la misma, puesto que aún son pocos los afiliados en la pequeña empresa y, cuando se ha puesto en marcha, ha sido derivando recursos, dinero y horas sindicales, conseguidos gracias a los afiliados y cuotas de otras empresas del sector. No obstante, los sindicatos son conscientes de que, si quieren conseguir penetrar en estas empresas y romper con el círculo vicioso que afecta a su acción y apoyos en ellas, han de llevar dicha estrategia hasta el límite de sus posibilidades.

Generalmente, la estrategia de penetrar en las pequeñas empresas se concreta también en el desarrollo de campañas de concienciación sindical de los trabajadores. Para ello, cuando es posible, hacen campañas de afiliación, que aprovechan para ofrecer información a los trabajadores sobre el convenio que los protege e, igualmente, para escuchar directamente sus demandas. En este sentido, los líderes sindicales están convencidos de que es necesario su presencia directa o, como ellos dicen, "el boca a boca (...) la relación personal", para que los trabajadores abandonen la idea de que los sindicatos sólo atienden a los problemas de los trabajadores de las grandes empresas.

\footnotetext{
${ }^{14}$ En España, la legislación laboral vigente concede a los representantes de los trabajadores un crédito de horas, dentro de la jornada de trabajo y sin merma del salario, para el desarrollo de sus funciones de representación. Dicho crédito de horas de los representantes se puede acumular en uno o varios de ellos pudiendo quedar liberados del trabajo. Para más información se pueden consultar la voces: Crédito de horas y Facilidades de los representantes de los trabajadores, en Martín Valverde, A. y J. García Murcia (1998), Glosario de Empleo y Relaciones Laborales, Madrid, Mundi-Prensa.
} 


\section{Incremento de los recursos económicos}

Para la implementación de las estrategias sindicales comentadas, los sindicatos necesitan incrementar y gestionar mejor sus recursos económicos, lo cual se convierte en otro objetivo sindical prioritario. De hecho, podemos encontrar tres estrategias diferentes con las que los dos grandes sindicatos, CC.OO. y UGT, han intentado alcanzarlo.

La primera, y principal, es la captación de afiliados. Los sindicatos argumentan que, actualmente, su principal vía de ingresos es la que consiguen con las cuotas sindicales. Además, consideran que si quieren aumentar su autonomía y capacidad de actuación, no constreñida por terceros, deben tratar de incrementar sus ingresos por esta vía y no por otras posibles, como las subvenciones estatales $o$, incluso, de las empresas.

“...desde el punto de vista de la financiación, el recurso más importante del sindicato son las cuotas y el servicio jurídico. Además otras financiaciones no creo que deban ser principales, no creo que sean buenas para la libertad sindical" (lider sindical regional de CC.OO).

En esta línea, persiguen, por una parte, incrementar el porcentaje de afiliados que poseen en aquellos sectores y empresas en los que ya están suficientemente asentados, y por otra, conseguir una masa crítica de afiliados y simpatizantes que les permita comenzar a desarrollar su labor en los que aún no lo están.

En relación con ello, tratan de desarrollar su faceta de empresarios políticos, ofreciendo incentivos selectivos a los trabajadores para que se afilien. En este sentido adoptan una racionalidad instrumental, dejando en segundo plano la sustantiva o de identidad. Sobre los incentivos selectivos que utilizan para incentivar la afiliación y acabar con la figura del "gorrón" (free-rider), hay algunos que se vienen utilizando de forma más generalizada que otros. Como ejemplos podemos citar la potenciación, allí donde es posible, de las secciones sindicales, como estructuras en las que sólo participan los afiliados y desde las que pueden participar directamente en el diseño y la priorización de los temas que se llevarán posteriormente a los comités de empresa ${ }^{15}$. También, la oferta de servicios, jurídicos y de otro tipo, gratuitos para los afiliados y con cierto coste para los no afiliados.

Asimismo y con objeto de aumentar la presencia sindical en las empresas en las que los sindicatos son prácticamente inexistentes, procuran especializarse en

\footnotetext{
${ }^{15}$ Con ello, además, resuelven el conflicto de competencias entre una estructura y otra. De forma que aunque el desarrollo de las negociaciones se haga utilizando el comité de empresa, la estrategia a seguir en el mismo se desarrollará en las secciones sindicales.
} 
sus problemas, e invirtiendo recursos, intentar conseguir el apoyo de este importante contingente de trabajadores, hasta ahora descreídos o desilusionados con un sindicalismo que se orientaba a la gran empresa.

La segunda estrategia puesta en marcha con objeto de incrementar los recursos sindicales, ha sido ampliar la oferta de servicios a sus afiliados. Con ello, los sindicatos pretenden ingresar dinero en sus arcas por dos vías. Por un lado, por la rentabilidad propia de convertirse en "empresa de servicios". Por otro, por el incremento de afiliados que se podría producir al ofrecer servicios exclusivamente para ellos a un precio competitivo. Los servicios que se han ofrecido han sido de lo más variado, desde la construcción de viviendas, hasta el revelado de fotos, pasando por conciertos con todo tipo de empresas para conseguir precios especiales o descuentos para sus afiliados. En general, la evaluación que los líderes sindicales hacen de dicha estrategia es negativa, al considerar que no ha servido para ingresar dinero de forma directa, ni tampoco de forma indirecta, puesto que tampoco ha repercutido claramente en el incremento de afiliados.

"[los servicios] no han sido un elemento importante para conseguir la afiliación, debe ser el trabajo diario el que lo consiga" (lider sindical de UGT).

"Los servicios en todo caso mantienen la afiliación pero no suponen una afiliación en masa (...) ayudan a mantener y a decidir a la afiliación pero no es lo fundamental, que es el trabajo día a día" (lider sindical de CC.OO).

No obstante, los servicios sindicales que siguen siendo más rentables y efectivos para la captación de afiliados, son los jurídicos y los relacionados con la formación de los trabajadores. Los sindicatos comenzaron a cobrar estos servicios a los no afiliados, con un doble objetivo. Por un lado, para no repercutir los gastos sobre los afiliados que pagan sus cuotas, y por otro, para demostrar a los no afiliados que dichas prestaciones tienen un precio de mercado y, si las quieren, o se afilian o las tienen que pagar.

La tercera y última estrategia para el saneamiento de la economía sindical y por ende para desarrollar estrategias que les permitan defender mejor los intereses de los trabajadores, ha sido abundar en la organización y mejor gestión de los recursos que poseen. Para ello, han creado o fortalecido estructuras que gestionen más ágilmente los archivos de afiliados y la recaudación de sus cuotas de forma directa, es decir, sin pasar por otras estructuras sindicales. Ejemplos de ello encontramos, tanto en CC.OO., como en UGT. En el primero, con la creación en 1991 de la U.A.R — Unidad de Administración y Recaudación. En el segundo, con el desarrollo de su Servicio Confederal Administrativo que, aunque fue creado en 1981, se consolidó plenamente en los 90.

Estos cambios estructurales y organizativos son un buen indicador de la importancia que tiene para los sindicatos españoles el objetivo del incremento y 
mejor gestión de sus recursos económicos, lo cual se entiende mejor cuando se conoce que, tanto el mantenimiento de un registro de afiliados fiable, como la correcta representatividad de las diferentes estructuras en los congresos y demás órganos sindicales de decisión, dependen, en gran medida, de la correcta organización de los ingresos por cuotas, que es el objetivo de los órganos anteriormente mencionados.

\section{Protección y promoción del empleo}

Otro objetivo sindical prioritario en este período para atender a los intereses de los trabajadores es la protección y promoción del empleo, es decir, la preocupación por la cantidad y la calidad del empleo. Para tratar de alcanzarlo los sindicatos españoles de los años 90 articulan diferentes estrategias. En primer lugar, a nivel de cúpulas, fomentan la negociación directa con el Gobierno, para todos aquellos temas relacionados con la política laboral, ya sea de forma sectorial o global. Con ello, pretenden ayudar a que se potencie, por una parte, el empleo, y, por otra, la estabilidad en el mismo. Ambos objetivos muchas veces se presentan como contradictorios, y la postura sindical ha sido intentar demostrar que la precarización de las condiciones laborales y de los contratos de los trabajadores no es una vía adecuada para potenciar el empleo. De hecho, el argumento de la mayoría de sus líderes es muy similar al que resume uno de ellos en la siguiente frase: “...en los últimos años, cada vez se ha flexibilizado y precarizado más el mercado de trabajo y eso no ha servido, en ningún caso, para rebajar las tasas de paro...". En coherencia con este argumento, los sindicatos intentan convencer a los empresarios y al Gobierno de la necesidad de utilizar otro tipo de vías para la promoción del empleo sin necesidad de precarizarlo. Entre ellas, las más enunciadas son: el reparto del empleo, es decir, la disminución de horas de trabajo para dar cabida a más trabajadores; la capitalización de las empresas, incluyendo en este concepto la formación y el reciclaje de sus trabajadores; $y$, sobre todo, la negociación con los sindicatos de todos aquellos temas relacionados con la flexibilización y la movilidad funcional y geográfica. Esta última opción sindical no es sólo una estrategia en favor de la estabilidad en el empleo, sino también el resultado de un importante cambio sindical. De hecho, años atrás, temas como la flexibilización o la movilidad eran innegociables $\mathrm{y}$, generalmente, motivo de multitud de conflictos allí donde los sindicatos tenían fuerza; y, por contra, algo consuetudinario, donde los sindicatos no llegaban con facilidad, como ocurría en las pequeñas empresas y sectores que no forman parte de la llamada industria clásica. Actualmente, como los sindicatos son conscientes de sus dificultades para defender esta postura en la mayoría de los sectores productivos y empresas, su estrategia se ha encaminado a promover que estos temas se negocien necesariamente con ellos, tanto a nivel nacional, como de sector y empresa. Con ello, pretenden controlar un fenómeno que, de 
no negociarse, podría provocar, y de hecho provoca, en muchos casos, la explotación de los trabajadores más indefensos, la competencia desleal y, en general, el deterioro de las condiciones laborales, del mercado de trabajo y de la economía.

Otra estrategia que los sindicatos tratan de poner en marcha, para la protección del empleo en los sectores o empresas que, aun contando con presencia sindical, la beligerancia patronal provoca que simplemente acercarse a los trabajadores pueda ponerlos en dificultades, es la de presionarlos para que faciliten a los representantes sindicales información sobre las condiciones laborales de cada uno de los trabajadores - a lo cual están obligados por ley ${ }^{16}$. De esta forma, vigilan indirectamente, es decir, sin implicar al trabajador en ello, que se cumpla lo legalmente estipulado. Con esto, los sindicatos intentan velar por los intereses de aquellos trabajadores que, por sus condiciones de inestabilidad, o por miedo, no recurren a los sindicatos. Además, consideran que con este trabajo conseguirán evitar situaciones de explotación o desigualdad encubiertas y, cuando dichos trabajadores estén en mejores condiciones laborales, tendrán más incentivo para afiliarse.

"cuando hay un trabajador nuevo, te presentas, le ofreces el convenio colectivo para que vea sus derechos, otras veces mandamos cartas a los nuevos, explicándoles su trabajo según contrato, le damos la dirección y nos ponemos a su disposición (...) pero los contratados temporales tienen tanto miedo que se lo adviertes: ' a partir de ahora no vuelvas a hablar conmigo en público'. No obstante tu vigilas su situación a través de los TC 1 y de la información que le puedes pedir a la empresa" (líder sindical UGT).

De forma parecida, los sindicatos tratan de articular estrategias, más o menos indirectas, para solucionar otros problemas como los que afectan a los trabajadores fuera de convenio. En este sentido adoptan una doble estrategia.

Por un lado, tratan de demostrar a los trabajadores de las empresas más proclives a esta nueva forma de negociación que, con toda probabilidad, las ventajas económicas que consiguen abandonando el convenio colectivo no son rentables, ni a corto ni a largo plazo. A corto plazo, porque el incremento salarial recibido no compensa el trabajo y la disponibilidad que implica dicha situación. Y, a largo plazo, porque se verán desprotegidos, y en situación muy desigual ante el empleador, para negociar correctamente sus condiciones.

\footnotetext{
${ }^{16}$ Ejemplo de ellas podemos encontrar en los artículos 64, 15.4, 19.5, 39.4, 39.2, 29.4, 41, 44.1, 51 en relación con el 47 del Estatuto de los Trabajadores, y otros en materia de contratación que les reconoce el artículo 1 de la ley 2/1991 sobre derechos de información de los representantes de los trabajadores en materia de contratación.
} 
Por otro lado, tratan de presionar a las empresas para convencerlas de que, de seguir profundizando en esta estrategia, los costes de negociación individual acabarán siendo superiores a sus supuestos beneficios: entre otros motivos porque la estrategia sindical se adaptará — de hecho ya se ha hecho en algún casoofreciéndole a los trabajadores la posibilidad de negociar por ellos con el empresario. De esta forma, los sindicatos homogeneizarán las demandas de los trabajadores, aunque en este caso por un camino indirecto.

No obstante, en España, el problema de los trabajadores con negociación individual o fuera de convenio afecta todavía a sectores muy concretos y, sobre todo, a trabajadores en puestos altos e intermedios de la estructura jerárquica de las empresas. Sin embargo, los grandes sindicatos españoles conocen que su supervivencia en el futuro pasa por integrar los intereses de este tipo de trabajadores, puesto que no sólo son más cada vez, sino que, o no se organizan colectivamente, o se organizan en sindicatos de cuadros o corporativos. En este sentido, no se han detectado estrategias claras de actuación sindical, aunque sí una toma de conciencia cada vez mayor de los problemas de estos trabajadores y, también, ciertos intentos de integrarlos y adaptar sus estructuras y servicios a los mismos ${ }^{17}$.

Otra estrategia que los sindicatos han desarrollado durante este período de tiempo para tratar de mejorar las condiciones laborales de los trabajadores es la negociación de convenios a diferentes niveles y la diversificación de las agendas de negociación, con temas diferentes a los salarios.

En cuanto a la negociación de convenios, los sindicatos siguen una doble estrategia. Por un lado, potencian la negociación de convenios, a nivel sectorial, estatal o autonómico. Por otro, potencian también los convenios de empresa o los de sector, a nivel provincial. Ello lo vienen haciendo con el objetivo de vencer las diferencias que se pueden dar entre las empresas de un mismo sector productivo, sin crear fuertes desigualdades. Por ejemplo, con el convenio sectorial superior, ya sea estatal o autonómico, pretenden establecer las condiciones mínimas que deben tener los trabajadores del sector y negociar aquellos temas relevantes a este nivel; $\mathrm{y}$, con los convenios de ámbito inferior, generalmente los de empresa, abren la posibilidad de adaptar y, en su caso, mejorar dichos mínimos, atendiendo a la naturaleza y características de las empresas en que se produzcan. Con esta estrategia, generalmente denominada negociación articulada,

\footnotetext{
${ }^{17}$ Un buen indicador de dicha toma de conciencia lo podemos encontrar en que en los foros de debate sindical el tema de la integración de los profesionales suele ser clave actualmente. Por ejemplo: en las Jornadas de debate que organizó UGT en 1998 con el título de "El futuro, la sociedad, el sindicato" de los siete foros de debate, uno se dedicó completamente al sindicalismo y los profesionales.
} 
pretenden resolver los problemas y desigualdades que se pueden dar, por ejemplo, entre los trabajadores de empresas pequeñas y grandes de un mismo sector productivo, protegiendo los mínimos deseables para todos sin por ello constreñir la posibilidad de mejora en las empresas donde sea posible.

Dicha estrategia se desarrolla paralelamente al intento sindical de que los convenios colectivos no se conviertan, exclusivamente, en acuerdos salariales. En este sentido, las organizaciones sindicales se están esforzando en demostrar a trabajadores y empleadores la conveniencia de negociar temas como la formación de la plantilla ${ }^{18}$, la flexibilidad en todas sus facetas, e incluso las posibilidades estratégicas del sector o la empresa.

En general, se debe destacar el espíritu negociador de las organizaciones sindicales que, conociendo los problemas laborales existentes en la actualidad y las condiciones del mercado de trabajo, se esfuerzan en agotar esta vía, antes de acudir a la utilización de la vía del conflicto. Se observa, incluso, el hecho de que la suspensión de una negociación en un determinado tema no haya sido óbice, en estos últimos años, para "levantarse de la mesa de negociación". Al contrario, en este caso, las organizaciones sindicales intentan agotar la vía negociadora, al menos, en los demás temas.

Las estrategias sindicales de presión también han sido objeto de cambios importantes durante los años 90. Los sindicatos, sin olvidar que la huelga es un derecho y una forma de presión fundamental, intentan poner en marcha otras vías de presión menos traumáticas para los trabajadores - muchos de ellos en precario-y, en ocasiones, más eficaces. Es difícil poner ejemplos concretos de cuáles son estas vías de presión, puesto que están muy vinculadas con el tipo de empresa o sector productivo en el que se desarrollan. No obstante, por lo general, están relacionadas con la utilización, en una forma u otra, de los medios de comunicación, con el cumplimiento estricto de la tareas o con cualquier otra maniobra con la que captar el apoyo de la sociedad o de los potenciales consumidores de los productos de la empresa con la que han entrado en conflicto.

\section{Evitar la confrontación intersindical}

Por último, destacaremos un objetivo que los sindicatos españoles consideran transversal, como es evitar la confrontación intersindical, ya sea por causa de

\footnotetext{
${ }^{18}$ Para desarrollar un estrategia en este aspecto, los sindicatos consideran que han sido de gran ayuda las subvenciones y ayudas otorgadas por el gobierno, u otras instituciones, a las empresas. Por ejemplo, el hecho de que los planes de formación que se presentan al FORCEM tengan que contar con el apoyo de los sindicatos, les permite estar informados y negociar con las empresas temas que, anteriormente, las últimas consideraban de su ámbito exclusivo
} 
las elecciones sindicales o las negociaciones de convenios de empresa, o por afinidad con algún partido político concreto.

En relación a la primera de las causas, las estrategias más desarrolladas son: dilatar siempre que sea posible, el espacio de tiempo entre los acontecimientos que provocan mayor confrontación y, sobre todo, potenciar cierta cultura de negociación intersindical previa. Todo ello, además, facilita no estar siempre implicados en procesos de negociación que conllevan mucho gasto de recursos económicos, materiales y humanos. Los sindicatos, actualmente, intentan que los convenios sean en lugar de anuales, bianuales, o incluso cada cuatro años. Todo ello siempre que sea posible, por un lado, introducir formalmente cláusulas para la revisión de aquellos temas que, como los salarios, así lo necesitan, y, por otro, plataformas estables para vigilar el desarrollo de lo acordado.

En relación a la segunda causa, la estrategia más desarrollada es, desde las cúpulas, potenciar la unidad de acción y la no división del movimiento obrero. En este sentido está ayudando bastante, según los propios líderes sindicales entrevistados, la desvinculación de los partidos políticos, lo que, de nuevo, vuelve a poner de manifiesto la creciente importancia de la racionalidad instrumental en el seno de las grandes organizaciones sindicales españolas y el paso a un segundo plano de la lógica ideológico-partidista o sustantiva que tan importante fue durante la transición política española.

\section{CONCLUSIONES}

Tras analizar el contexto de la situación, los intereses de los trabajadores españoles de finales del siglo $\mathrm{XX}$ - a través del análisis de los problemas que consideran que tienen y las demandas que dirigen a los sindicatos- y los principales objetivos y estrategias de los sindicatos españoles, la principal conclusión que se puede alcanzar es que los grandes sindicatos españoles, UGT y CC.OO, están adaptándose estructural y funcionalmente para defender los principales intereses de los trabajadores de los años 90. Ello significa, al menos, que la detección de necesidades y de demandas de los trabajadores que realizan los grandes sindicatos españoles, así como la evaluación de sus propias necesidades para adaptarse a aquéllas, las han realizado con eficacia razonable. Esto se manifiesta tanto en el discurso de sus líderes, como en el diseño de la mayoría de sus estrategias, tal como antes se ha expuesto.

No obstante, esto no implica que tales estrategias hayan de ser necesariamente efectivas. Ello sólo podrá comprobarse con la observación de sus resultados en los años venideros. Es decir, evaluando en qué medida las estrategias implementadas por los sindicatos se traducen, efectivamente, en el mantenimiento o mejora de la estabilidad y las condiciones laborales de los trabajadores - sus principales intereses actualmente-; en el incremento de la afiliación y de los 
recursos sindicales - principales necesidades de los sindicatos- o en la promoción de la cantidad y de la calidad del empleo, especialmente de aquellos grupos que se ha comprobado que tienen más problemas para integrarse en el mundo laboral contemporáneo: mujeres y jóvenes.

No obstante, la incertidumbre sobre los resultados de las estrategias puestas en marcha por los grandes sindicatos españoles de finales de siglo no es suficiente para considerar que éstos están en crisis o son obsoletos, mucho menos cuando la información empírica que se ha analizado demuestra, por un lado, que sus objetivos responden a los principales intereses de los trabajadores y, por otro, que están desarrollando estrategias y acciones coherentes con el contexto en el que actúan y con su propia situación organizativa para alcanzarlos con éxito.

En suma, con lo expuesto no se trata de justificar que los sindicatos españoles hayan alcanzado su nivel máximo de adaptación y respuesta a los intereses de los trabajadores de final de siglo. Al contrario, se apunta la idea de que aún les falta mucho camino por recorrer, sobre todo, en su proceso de adaptación, defensa y oferta de alternativas a aquella parte de la población que más problemas tiene para encontrar empleo. Sin embargo, sí se considera suficiente para concluir con cierto grado de verosimilitud, contestando a la pregunta que se hacía al principio de este trabajo, que el sindicalismo español de finales de siglo no está obsoleto y, por ende, que la tesis de la crisis u obsolescencia de las organizaciones sindicales en las sociedades industriales avanzadas, al menos, en su formulación de inadaptación e incapacidad de éstas para la defensa de los intereses de los trabajadores, no se demuestra para el caso del sindicalismo español en las postrimerías del siglo $\mathrm{XX}$.

\section{BIBLIOGRAFÍA}

AGUILAR, S. y J. ROCA (1991), Trabajadores y sindicatos en una sociedad compleja. Las estrategias de los sindicatos españoles durante la transición. Mimeo, ponencia presentada al seminario "La integración sindical en estructuras complejas", Sevilla, UIMP.

BLANCO, J. (1991), "La sindicación de trabajadores de pequeñas y medianas empresas", Sociología del Trabajo, num. 11.

ESCUDERO, R. (1990), La representatividad de los sindicatos en el modelo laboral español, Madrid, Tecnos.

FERNER, A. y R. HYMAN (eds.) (1998), Changing Industrial Relations in Europe, Oxford, Blackwell.

FISHMAN, R. (1996), Organización obrera y retorno de la democracia en España, Madrid, CIS.

FOESSA (1994), V Informe sociológico sobre la situación social en España. Sociedad para todos en el año 2000, Madrid, Fundación FOESSA. 
FREEMAN, R.B. y J.L. MEDOFF (1984), What do Union Do? United States of America, BasicBooks. A division of collins publishers.

FÜHRER, I. M. (1996), Los sindicatos en España. De la lucha de clases a estrategias de cooperación, Madrid, Consejo Económico y Social.

GOETSCHY, J. (1998), "France: The Limits of Reform", Ferner, A. y R. Hyman (ed.) Changing Industrial Relations in Europe, Oxford, Blackwell.

GALlIE, D., M. ROSE, et al., (1996), The British Trade Unionism: Crisis and Continuity. Trade Unionism in Recession, Oxford, Oxford University Press

INSTITUTO DE ESTUDIOS y ANÁLISIS ECONÓMICOS (1993), Sindicatos, economía y sociedad: un futuro para el sindicalismo español, Madrid, Ministerio de Economía y Hacienda.

JORDANA, J. (1994), "Sindicatos y política en España: la influencia de las condiciones organizativas en la estrategia sindical", Revista Internacional de Sociología, 8-9: 137-188.

LAWLOR, T. y M. RIGBY (eds.) (1998), Contemporary Spain. Essays and texts on politics, economics, education and employmenty, and society, London \& New York, Longman.

LEISINK, P., J. VAM LEEM, et al., (eds.) (1996), The Challenges to Trade Unions in Europe, Cheltenham, Edward Elgard.

MARTÍN VALVERDE, A. y J. GARCÍA MURCIA (1998), Glosario de Empleo y relaciones laborales, Madrid, Mundi-Prensa Libros, S.A.

MIGUEL, A. de (1992), La sociedad española 1992-93, Madrid, Alianza

MCILROY, J. (1995), Trade Unions in Britain Today, Manchester, Manchester University Press.

PANEBIANCO, A. (1990), "Los partidos y la democracia: institucionalización y crisis", Modelos de Partido. A. Panebianco, Madrid, Alianza Universidad.

PÉREZ YRUELA, M. (1998), "El sindicalismo y los profesionales", El futuro, la sociedad, el sindicato. C. E. F. UGT, Madrid, Publicaciones Unión, UGT.

REGINI, M. (ed.) (1994), The Future of Labour Movements, London, SAGE /ISA.

RIGBY, M. y R. SERRANO DEL ROSAL (eds.) (1997), Estrategias Sindicales en Europa: Convergencias o Divergencias, Madrid, Consejo Económico y Social.

RIGBY, M., R. SMITH, et al. (eds.) (1999), European Trade Unions: Change and Responses, London, Routledge.

SANCHIS, E. (1991), De la escuela al paro, Madrid, Siglo XXI.

UNDY, R., P. FOSH, et al. (1996), British Trade Unions Strategies in a Hostile Enviroment 19801993. The Challenges to Trade Unions in Europe, Cheltenham, Edward Elgar. 
VISSER, J. (1992), "The Strengh of Unions Movements in Advanced Capital Democracies: Social and Organizational Variations", Regini, M.(ed.), The Future of Labour Movements, SAGE, ISA, London.

WEBB, S. y B. WEBB (1990), Historia del sindicalismo, Madrid, Ministerio de Trabajo y Seguridad Social.

ABSTRACT. In this article, the author discuss the thesis of the crisis or obsolescence of unionism in advanced industrial societies by analyzing the Spanish case at the turn of the 20th century. The article first addresses recent developments within the Spanish labor market to describe how workers define their problems and pose their demands, and how labor unions articulate their goals and tactics to comply with, and to defend workers' interests. Secondly, it discusses the workers' interests, that is, the problems envisioned by the workers as well as their particular demands to the unions. And third, it portrays the unions' main goals and their specific strategies to achieve these goals. Finally, with all these elements in mind, the author will address the question of whether or not, in the context of the late 20 th century, Spanish labor unions adapt to the workers' interests.

E-mail: rserrano@iesaa.csic.es 